\title{
DEVELOPMENT OF FLEXIBLE, CONDUCTIVE AND BIOCOMPATIBLE CHITOSAN-BASED MINIATURIZED BIOELECTRODES FOR ENZYMATIC GLUCOSE BIOFUEL CELLS
}

\author{
Laura García-Carmona $^{1 *}$, Mireia Buaki-Sogó ${ }^{*}{ }^{*}$, Marta Vegas-García ${ }^{1}$, Mayte Gil-Agustí ${ }^{1}$, Pedro Llovera-Segovia ${ }^{2}$, Alfredo \\ Quijano-López ${ }^{1,2}$. \\ ${ }^{1}$ Instituto Tecnológico de la Energía (ITE), Avenida Juan de la Cierva 24, 46980 Paterna, Spain; \\ ${ }^{2}$ Instituto de Tecnología Eléctrica, Universitat Politècnica de València, Camino de Vera s/n Edificio 6C, 46022 Valencia, Spain
}

\begin{abstract}
The need for new clean energy sources for portable devices in biomedical, agro-food industry and environmental related sectors boosts scientists towards the development of new strategies for energy harvesting for their application in biodevices development. In this sense, enzymatic biofuel cells (BFCs) have gained much attention in the last years. This work faces the challenge of develop new generation of BFCs able to be adapted to remote and personal monitoring devices within the framework of wearable technologies. To this aim, one of the main challenges consists of the development of conductive and biocompatible electrodes, which constitute a challenge itself due to the non-conductive capabilities of most of the biocompatible supports. Additionally, bioelectrodes may achieve good mechanical properties and resilience in order to be suitable for the envisioned application, which involves exposure to deformation during long-term use. Furthermore, it is desirable that the systems developed are versatile enough to be adapted to miniaturized supports for new personal wearable devices development. In the present work, selfstanding chitosan-carbon black membranes have been synthesized and modified with suitable enzymes for the assembly of an enzymatic glucose BFC. The membranes have been adapted to be integrated in miniaturized interdigitated gold electrodes as the step forward to miniaturized systems, modified with enzymes and metallic particles clusters and tested for energy harvesting from glucose solutions. The miniaturized system produces a power density of $0.64 \mu \mathrm{W} / \mathrm{cm} 2$ that is enhanced to $2.75 \mu \mathrm{W} / \mathrm{cm} 2$ in the presence of the metallic clusters, which constitute a $76 \%$ incensement. Such preliminary demonstrations highlight the good response of metals in bioelectrode configuration. However, energy harvesting real application of the developed miniaturized electrodes need still improvements but pave the way for the use of $\mathrm{BFC}$ as an energy source in wearable technologies due to their good mechanical, electrical and biocompatible

properties.
\end{abstract}

\section{INTRODUCTION}

Chitosan is a natural biopolymer obtained from the deacetylation of chitin, which can be found, for example, in shrimp shells. Chitosan has been widely used in the development of enzymatic bioeletrodes due to its capability for stabilising enzymes in the biopolymer network, hydrophilicity and biocompatibility [1][2][3]. Nevertheless, chitosan presents an insulating behaviour and need to be integrated with conducting surfaces or doped with conducting agents when developing enzymatic bioelectrodes. Thus, the main strategy in the use of chitosan to be used in enzymes immobilization consists of incorporating the enzyme in a solution of chitosan and then, cast it on the surface of common electrode such as graphite, glassy carbon or gold electrodes[4][5]. Additionally, it is usual to dope chitosan with carbon-based materials such as carbon nanotubes, graphene or carbon black to overcome the insulating barrier [4][5][6].

In this sense, the former approach of casting onto the surface of electrodic materials is useful when dealing with bioelectrodes to be used in biosensor development with analytical purpose or electrochemical characterization of enzymes and their bioelectrocatalytic activity. However, when dealing with enzymatic glucose biofuel cells (BFC) for energy harvesting aimed to be wearable or implantable, new strategies must be implemented since long-term use is required.

Glucose BFCs consist of biodevices aimed to harvest energy from human body due to the catalytic electrochemical oxidation of glucose as a result of the selective recognition of a substrate (glucose) by the enzyme, typically glucose oxidase (GOx) or glucose dehydrogenase (GDH). Thus, low cost and availability of the enzymes are required, together with stable immobilization methods on the surface such as crosslinking, entrapment, covalent anchorage to allow stability of the binding for the long term use [7].

However, advances in wearable technologies need for new electrode configurations that can be placed anywhere in the body. For this reason, miniaturization strategies are of interest for the development of small prototypes of such biodevices. In this sense, chitosan has the ability of forming films that could be used as electrodes in the form of a path or that could be integrated in existing systems such as screen-printed, interdigitated electrodes or in flexible electronics [4][5][8][9]. Thus, chitosan thanks to

Corresponding authors: mireia.buaki@ite.es (M.B.-S.) and laura.garcia@ite.es (L.G.-C.); Tel.: +36-9-6136-6670. 
their biocompatibility characteristics and its film forming ability has been used to develop a biopolymeric membrane of chitosan doped with carbon black that presents the capability of acting as a support for enzyme immobilization and as self-standing electrode at the same time [1]. The chitosan-carbon black membrane with $25 \%$ $\mathrm{w} / \mathrm{w}$ or $100 \% \mathrm{w} / \mathrm{w}$ with respect to chitosan can be integrated in the most convenient configuration using additional conductive layers. However, taking into account the miniaturization and further use in flexible electronics, this procedure already tested for glucose BFCs and biosensor should be optimized to be integrated into miniaturized supports with conductive layers for connection already included in the support.

On the other hand, either in film or miniaturized form, chitosan-carbon black based electrode can be doped and modified using electrochemical methods for metals electrochemical synthesis in its surface. In this sense, different electrochemical methods have been used for metal deposition [10]. Specifically, double pulse potential constitutes a very convenient method since it is possible to create clusters in situ in a distributed way all along the electrodic surface. As its name suggest, two pulses are applied to the cell in which the first one (in the order of $\mathrm{ms}$ ) is responsible of the generation of nuclei of the particles, meanwhile the second pulse (in the order of s) allow the growth of those particles [11]. This is a highly valuable synthesis feature since metals included in BFCs configuration have been proved to enhance the power input by improving the electron transfer rate [12].

Thus in this work, the chitosan-carbon black membrane composition have been optimized to be integrated into miniaturized supports to be adapted to real systems and later on tested to be used in glucose BFCs. In this context, integration of metallic clusters on the membrane surface have been tested to evaluate the feasibility of the membranes to be in situ modified but also the possible enhancement in the response of the BFCs as prove of the concept of this approach.

\section{EXPERIMENTAL SECTION}

\subsection{Materials}

Chitosan of medium molecular weight with a deacetylation degree higher than 85\% (Sigma-Aldrich. St. Louis, MO, USA), glacial acetic acid reagent grade, $\mathrm{NaOH}$ (Scharlab, S.L.,Barcelona, Spain), and C65 Carbon black powder (Nanographi, Ankara, Turkey) were used for the preparation of membranes using water as solvent.

Glucose oxidase (GOx) from Aspergillus niger Type VII and Laccase from Trametes versicolor were used for bioelectrodes preparation (Sigma-Aldrich (St. Louis, MO, USA)). N-(3-dimethylaminopropyl)-Nethylcarbodiimide (EDC) and Nhydroxysulfosuccinimide (NHS) were btained from Iris Biotech Gmbh (Marktredwitz, Germany), and SigmaAldrich (St. Louis, MO, USA), respectively. 2-(Nmorpholino)ethanesulfonic acid for the MES buffer and D-Glucose (biotechnology grade) were provided by
VWR LIFE SCIENCE (Radnor, PA, USA)., $\mathrm{Na}_{2} \mathrm{HPO}_{4}$ and $\mathrm{NaH}_{2} \mathrm{PO}_{4}$ were acquired from VWR LIFE SCIENCE (Radnor, PA, USA for the preparation of $0.1 \mathrm{M}$ Phosphate Buffer solutions $\mathrm{pH}=7$.

Gold interdigitated electrodes (IDE) from Micrux Technologies were employed as a miniaturized electrodes support.

\subsection{Preparation of chitosan membranes}

Self-standing chitosan membranes doped with carbon black were prepared as following a Doctor Blade strategy by way of a stainless steel casting knife protocol previously reported [1]. In brief, slurry was first prepared by mixing an appropriate chitosan solution with carbon black. The slurry was casted onto a glass surface with the aid of a casting knife and dried overnight. After a neutralization step with $\mathrm{NaOH}$ solution was accomplished, followed by different washing steps up to reach neutral $\mathrm{pH}$. The as-prepared membranes were allowed to dry onto a flat surface overnight.

\subsection{Modification of interdigitated electrodes}

Gold interdigitated electrodes from Micrux Technologies $(10 \times 6 \times 0.75)$ were modified with chitosan-carbon black membranes. At this purpose, slurry composition was optimized in order to ensure that the chitosan-carbon black membrane remains into the electrode surface to ensure long-term use of the bioelectrode.

For the immobilization of GOx, the interdigitated electrodes were modified with a membrane of chitosanCB. The composition of the slurry was a chitosan solution of $1 \% \mathrm{w} / \mathrm{w}$ in acetic acid $0.3 \mathrm{M}$ with $50 \% \mathrm{w} / \mathrm{w}$ of carbon black. The mixture was prepared in a planetary ball mill and after a volume of $15 \mu \mathrm{l}$ of the slurry was casted on the gold surface of interdigitated electrodes.

For the immobilization of Laccase, the slurry composition is based on a solution of chitosan $2 \% \mathrm{w} / \mathrm{w}$ doped with $25 \% \mathrm{w} / \mathrm{w}$ of carbon black with respect to chitosan. As in the case of modified interdigitated electrodes for the anode, a planetary ball mill was used for mixing the components and the same volume of $15 \mu \mathrm{l}$ was casted onto the gold surface of the interdigitated electrode.

The incorporation of metallic clusters in order to enhance electrode response was accomplished electrochemically by double pulse potential voltammetry (1 sg for nucleation potential and $10 \mathrm{sg}$ for grow potential) in $\mathrm{AgNO}_{3}$ solution. Then, bioelectrodes were electrochemically oxidized using $\mathrm{NaOH} 0.1 \mathrm{M}$ at $0.7 \mathrm{~V}$ during $120 \mathrm{sg}$.

\subsection{Enzyme immobilization}

GOx and Laccase were immobilized in the membranes for bioelectrode development. For the GOx-based anode $10 \mathrm{mg} / \mathrm{mL}$ GOx was immobilized in the $\mathrm{CH}-\mathrm{CB}$ membranes using a covalent approach based on the use of EDC/NHS as previously reported [1]. In the case of Laccase, the enzyme was first adsorbed onto the $\mathrm{CH}-\mathrm{CB}$ starting from a $10 \mathrm{mg} / \mathrm{mL}$ solution of Laccase in $\mathrm{PB} \mathrm{pH}$ 
6.5. For the covalent anchoring to the amino groups of the chitosan matrix, an approach based on the use of EDC/NHS was also used based on a procedure previously reported [1][13].

\subsection{Electrochemical testing}

An Autolab PGSTAT 12 potentiostat (Eco Chemie, Utrecht, The Netherlands) and a Zhaner IM6 electrochemical workstation provided with a PP241 module for high currents was used for cyclic voltammetry, chronoamperometry, double pulse potential voltammetry and BFC polarization curve determination. Electrochemical characterization of the GOx bioanode, Laccase biocathode were performed in a three electrode configuration using developed miniaturized bioelectrodes as the working electrode, a reference electrode of $\mathrm{Ag} / \mathrm{AgCl}$, and a platinum wire as the counter electrode under $\mathrm{O}_{2}$ or $\mathrm{N}_{2}$ purged PBS buffer solution.

\section{RESULTS AND DISCUSSION}

\subsection{Miniaturization of chitosan $C B$ membrane for bioelectrodes development.}

Chitosan-carbon membrane can be prepared using different carbon nanomaterials. However, CB has been selected due to their conductive properties and tuneable surface. The chitosan-CB membrane final structure will vary depending on whether it is a film or if it is intended for integration in miniaturized supports. In case of selfstanding membrane development (Figure 1A) the composition has been selected according to the resilience to mechanical strain stretching, bending, and twisting, together with its the conductivity properties [1]. However, when adapted to miniaturized supports (figure 1B) this composition should be optimized to avoid detachment from the support. In this sense chitosan - CB ratio is crucial to be optimized to find a compromise between chitosan attachment properties and conductivity capabilities thanks to $\mathrm{CB}$.

The composition of the original self-standing membranes, with a $25 \% \mathrm{w} / \mathrm{w}$ and $100 \% \mathrm{w} / \mathrm{w}$ of $\mathrm{CB}$ for cathode of anode respectively, resulted in the detachment of the film from the gold interdigitated electrode in the case of the anode configuration. The film formed onto the gold surface of the IDE for cathode configuration remained attached onto the gold surface. To diminish the solid content on the membrane and prevent membrane folding and detachment an anode configuration with a $50 \% \mathrm{w} / \mathrm{w}$ of $\mathrm{CB}$ was checked. In this case membrane detachment was also observed.

In case of the anode, the reason for not using the same configuration as the cathode is that carbon black is oxidized to generate carboxylic acid groups that will be used later as anchoring site for the GOx. The Laccase anchorage follows a different strategy using amino groups of chitosan for its covalent attachment on the membrane surface. Thus, the role of $\mathrm{CB}$ in this configuration is to overcome the insulating behaviour of chitosan films. Therefore, it is better if CB particles are homogeneously distributed in the chitosan matrix and, as we observed in our previous work, this occurs for $\mathrm{CB}$ weight percentages of $50 \%$ or higher, when polymeric matrix is not visible at low magnification [1].

At this point, the next step for the optimization of the anode configuration in gold interdigitated electrodes was the decrease of the concentration of the chitosan solution in order to decrease film thickness and prevent detachment. When chitosan concentration was decreased to $1 \% \mathrm{w} / \mathrm{w}$ in the acetic acid solution, the membrane detachment was observed for $\mathrm{CB}$ weight percentages higher than $50 \%$. Therefore, the anode configuration selected for the integration of the chitosan-CB membranes in the miniaturized electrodes was $1 \%$ weight percentage for the chitosan solution in acetic acid, doped with a $50 \% \mathrm{w} / \mathrm{w}$ of $\mathrm{CB}$ with respect to chitosan.

\subsection{Self-standing chitosan membrane bioelectrodes for BFC development.}

The self-standing chitosan-CB bioelectrodes already modified with the enzymes were tested by cyclic voltammetry in order to confirm the presence of the immobilized enzyme for anode (Glucose Oxidase) and cathode (Laccase) on the membrane surface (Figure 2). In the cyclic voltammetry experiments carried out in $\mathrm{N}_{2}$ purged atmosphere for the membrane based bioanode, it can be observed the presence of the oxidation and reduction peaks of the redox centre (Flavin Adenine Dinucleotide - FAD) of the glucose oxidase enzyme. The formal potential of the redox peaks was $0.45 \mathrm{~V}$ in accordance with the values found in literature.
A

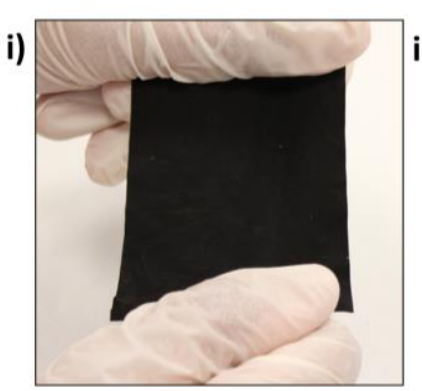

ii)

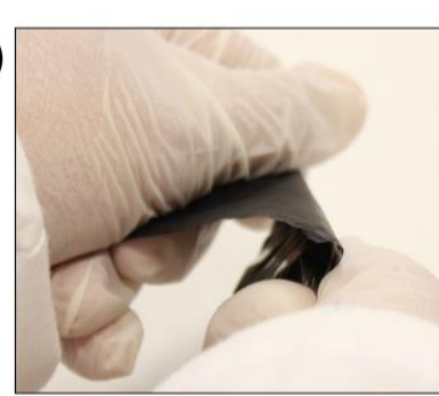

B

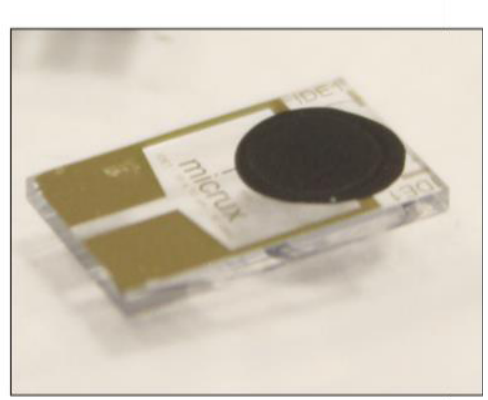

Figure 1. Chitosan-CB membrane configuration such as (A) self-standing membrane under (i) stretching and (ii)) twisting, and (B) membrane integrated into miniaturized electrodes. 
A

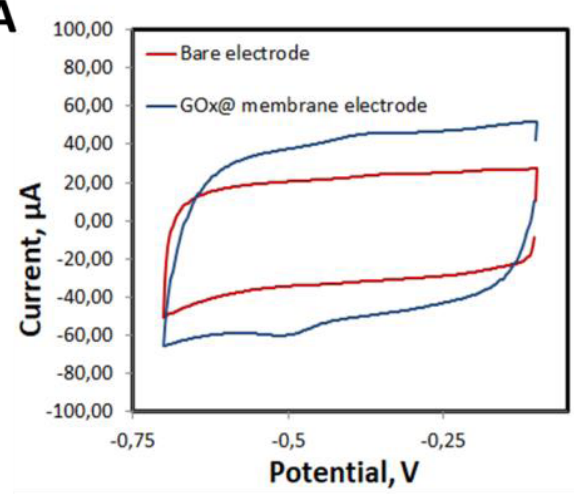

B

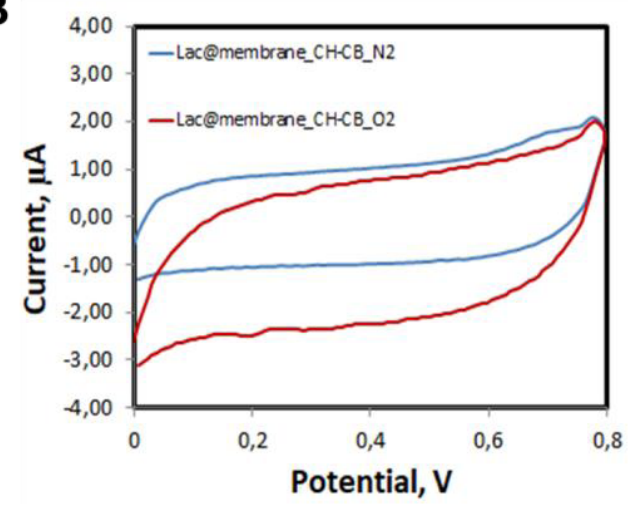

Figure 2. Bioelectrodoes evaluation in terms of enzyme attachment for, (A) GOx bioanode and (B) Laccase biocathode. Cyclic voltammetry in $\mathrm{PBS} 0,1 \mathrm{M}$ and $\mathrm{KCl} 0,1 \mathrm{M}$ in $\mathrm{N}_{2}$ saturated solution (bioanode) and $\mathrm{N} 2 / \mathrm{O} 2$ saturated solution (biocathode). Scan rate: $75 \mathrm{mV} / \mathrm{s}$

The presence of Laccase on the chitosan-CB membrane that will be used as the cathode was ascertained using also cyclic voltammetry in the presence of oxygen and the appearance of an onset around $0.7 \mathrm{~V}$ for the reduction of oxygen confirmed the successful immobilization of Laccase. However, and as observed in our previous work, the onset and the current generated are of low magnitude probably due to the working electrolyte at $\mathrm{pH} 7$, far from the optimum working $\mathrm{pH}$ reported for Laccase $(\mathrm{pH}$ 4.2) [14]. It is worth to mention that acid $\mathrm{pH}$ is not used since at that $\mathrm{pH}$ chitosan will become protonated and the membrane will be dissolved. Additionally, the BFCs are envisioned to be used in wearable technologies where a physiological $\mathrm{pH}$ is expected.

In order to evaluate the suitability of the developed selfstanding bioelectrodes for energy harvesting from glucose solutions a BFC was assembled in a lab scale using the GOx based bioelectrode as anode and the Laccase bioelectrode as cathode. Polarization curve was recorded and power and current density was calculated from the results of the polarization experiment. The area used for the calculation of power and current density was the electroactive surface area obtained from RandlesSevcik experiments for the miniaturized electrodes. The results for the polarization curve are showed below. Prior to bioelectrodes miniaturization, the geometric area of the electrodes employed was calculated as $0.6 \mathrm{~cm}^{2}$ [1] achieving thus an electroactive area of $1.2 \mathrm{~cm}^{2}$. After the miniaturization procedure into gold interdigitated electrodes, the electroactive area was reduced to $0.03 \mathrm{~cm}^{2}$ compared with the electrodes used in previous work using non miniaturized suppors with additional conductive layers for connections [1].

In this sense, Figure 3 shows the polarization curves obtained by the complete configuration of glucose BFC using non miniaturized bioelectrodes from [1], with maximum power density of $62.7 \mu \mathrm{W} / \mathrm{cm}^{2}$ at $0.19 \mathrm{OCV}$. It proves the successful enzyme immobilization technique and the retention of the specific electrocatalytic activity of the immobilized enzymes.

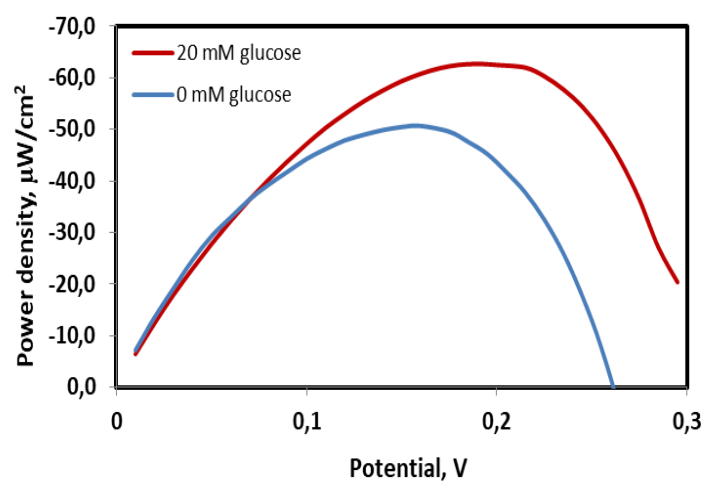

Figure 3. Glucose BFC power density (GOx-based anode and Laccase-based biocathode) in PBS $0.1 \mathrm{M}$ (blue) and $20 \mathrm{mM}$ glucose (red). Results obtained in larger electrodes, before miniaturization procedure $\left(0.6 \mathrm{~cm}^{2}\right)$.

\subsection{Integration of chitosan membranes in gold interdigitated electrodes}

In order to integrate the developed membranes in real and miniaturized systems, the chitosan-based membranes have been incorporated in interdigitated gold electrodes. Onto these membranes, following the same immobilization strategy stated and proved above, GOx and Laccase have been incorporated on the modified interdigitated electrodes. The polarization curve has been recorded and the results obtained are shown in Figure 4.

In this case, power densities obtained are lower than those recorded with the self-standing bioelectrodes. This could be due to the fact that geometrical and electroactive surface area of the interdigitated electrodes is lower and therefore current generated in the BFC assembly is decreased. However, miniaturized electrodes required to be equally efficient in terms of energy harvesting to be used to power monitoring devices. Then, in these systems it will be necessary to enhance the electrode response to increase the power generation in miniaturized systems.

To this aim, metallic clusters on the electrode surface have been in situ synthetized into the anode in order to improve electron transfer kinetics and bioelectrode response using the double pulse potential technique

\footnotetext{
Corresponding authors: mireia.buaki@ite.es (M.B.-S.) and laura.garcia@ite.es (L.G.-C.); Tel.: +36-9-6136-6670.
} 
[11][1]. As it has been already explained, this method allows direct silver electro synthesis in the bioelectrodes in less than 1 minute. Additionally, this technique creates nuclei of the particles in the first pulse in a distributed way all along the electrode surface than, later on growth during the second pulse. This fact lead to a distributed and homogeneous particles disposition in the membrane that will benefit electron transfer kinetics.

Energy harvesting capabilities were evaluated to test GOx activity and the suitability of $\mathrm{CH}-\mathrm{CB}$ membrane modified with silver metallic clusters. Figure 4 shows the comparison between the complete BFC composed by anode that incorporates metallic clusters on the configuration (purple line) and a bare electrode, containing just the enzymatic component (green line) with a characteristic bell-shaped curve. The biocathode employed in this study was a Laccase-modified bioelectrodes for both anode configurations. From this, it can be noticed that the maximum power density output is obtained with the silver-base anode is used, reaching a value of $2.75 \mu \mathrm{W} / \mathrm{cm}^{2}$ at $0.2 \mathrm{~V}$ which represents an increase of the power density in $76 \%$ with respect to that reached without metallic cluster in the setup.

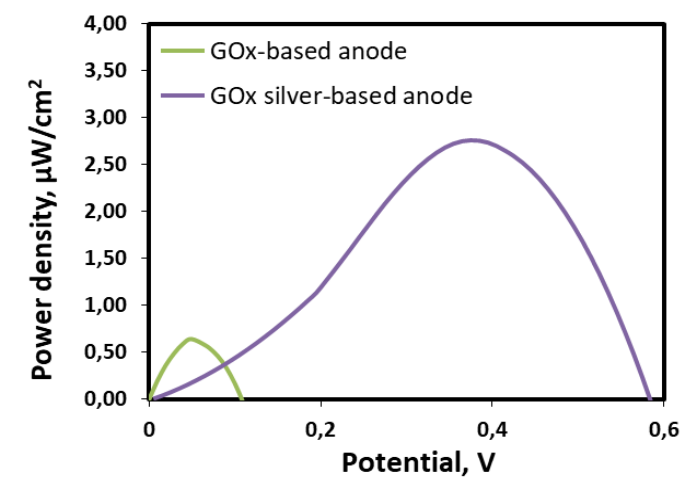

Figure 4. Glucose BFC polarization curves using two different anodes GOx-bioanode (green) and GOx-Ag particles-bioanode (purple). Laccase-based biocathode has been used in both cases. $25 \mathrm{mM}$ glucose has been used as fuel in both cases (TP 0.1M, using gold interdigitated electrodes).

This result shows the good feasibility of the membrane to be integrated in different supports such as interdigitated electrodes. Additionally, this results suggest the good electrocatalytic behaviour of the metallic particles, due to the performance of $\mathrm{Ag}_{2} \mathrm{O}$ to reduce $\mathrm{O}_{2}$, paving the way to a new trend of the incorporation of metallic elements on the bioelectrodes. However, these density power outputs values should be large increased for real application in wearable technologies. In this sense, it is foreseen the connection of several miniaturized electrodes in serial in case the final application requires maximum power inputs.

\section{CONCLUSIONS}

In this work it has been reported the development of a biocompatible membrane that can be miniaturized maintaining good mechanical and electrical properties at the same time. This shape- and size-tailored membrane, allows its deposition in a variety of substrates such as films or rigid supports, enabling the versatility to be adapted to any design for use in multiple applications such as the production of wearable devices with flexible electronics. Moreover, its suitability to be used in the development of bioelectrodes for glucose BFCs haven been proved thanks to the positive results obtained in energy harvesting for larger electrodes $\left(62.7 \mu \mathrm{W} / \mathrm{cm}^{2}, 0.6\right.$ $\mathrm{cm}^{2}$ ). In this regard, and despite to the low density power output obtained with the miniaturized electrodes, an improvement of energy harvesting capabilities have been obtained by means of the incorporation of metallic particles on the bioanode, producing an increase of the final power density in a $76 \%$ when compares to the bareelectrode setup. It is also important to mention that Lacasse-based cathode is used at $\mathrm{pH} 7$ which is not the optimal working condition of this enzyme.

Additionally, this development paves the way for the use metals in the anode configuration but also foresee the use of hybrid BFC, with enzymatic bioanode and metal-based cathode, to solve the issue of Lacasse-based electrode at physiological $\mathrm{pH}$. However, more research is required in terms of electronics, in order to develop higher power output energy harvesting strategies.

\section{ACKNOWLEDGEMENTS}

This work has been supported by the Generalitat Valenciana towards Instituto Valenciano de Competitividad Empresarial (IVACE) in accordance with the IMAMCL/2020/1 agreement and within the framework of the BioSensCell and BioCell-Power projects.

\section{REFERENCES}

[1]. M. Buaki-Sogó, L. García-Carmona, M. Gil-Agustí, M. García-Pellicer, A. Quijano-López, Nanomaterials $11(2021)$

[2]. S. Haque, A.Nasar, Inamuddin, M. Muzibur. Sci Rep 10, 10428 (2020)

[3]. S. El Ichi, A. Zebda, J.-P Alcaraz, A. Laaroussi, F. Boucher, J. Boutonnat, N. Reverdy-Bruas, D. Chaussy, M.N. Belgacem, P. Cinquin, D.K. Martin, Energy Environ. Sci., 8, 1017-1026, (2015).

[4]. D. Talarico, F. Arduini, A. Amine, I. Cacciotti, D. Moscone, G. Palleschi, Anal Bioanal Chem 408, 7299-7309 (2016)

[5]. A. Rabti, W. Argoubi, N. Raouafi, Microchim Acta 183, 1227-1233 (2016)

[6]. V. Mani, M. Govindasamy, SM. Chen, TW. Chen, A. Sentil Kumar, ST. Huang, Sci Rep 7, 11910 (2017)

[7]. M. Buaki-Sogó, L. García-Carmona, M. Gil-Agustí, L. Zubizarreta, M. García-Pellicer, A. Quijano-López, Top. Curr. Chem., 378, 1-28, (2020)

[8]. U. Hashim, M.A. Farehanim, N. Azizah, S. Norhafiezah, M.F. Fatin, A.Rahim Ruslinda, R.M. Ayub, IEEE ICoBE, pp. 1-3, (2015)

[9]. P. Wang, M. Hu, H. Wang, Z. Chen,Y. Feng, J. Wang, W. Ling, Y. Huang, Adv. Sci. , 7, 2001116, (2020)

[10].Y.Tang, W. Cheng, Langmuir, 29(9), 3125-3132 (2013). 
[11]. L. García-Carmona, D. Rojas, M.C. González, A. Escarpa, Analyst, 141(21), 6002-6007, (2016).

[12]. F.Qu, X. Ma, Y. Hui, F. Chen, Y. Gao, Chin. J. Chem. 35(7), 1098-1108 (2017).

[13]. C. Gutiérrez-Sánchez, W. Jia, Y. Beyl, M. Pita, W. Schuhmann, A.L. de Lacey, L. Stoica, Electrochim. Acta, 82, 218-223 (2012)

[14]. B. Reuillard, C. Abreu, N. Lalaoui, A. Le Goff, M. Holzinger, O. Ondel, F. Buret, S. Cosnier, Bioelectrochemistry, 106, 73-76 (2015) 